\title{
Deutschland 2020: unheilbar gespalten?
}

\section{Anmerkungen zur Ost-West-Differenz im 30. Jahr der Wiedervereinigung}

\author{
Everhard Holtmann
}

\section{Unterschiedlich, ungleich, aber auch konvergent - Ost- und Westdeutschland im Spiegel ausgewählter Struktur- und Einstellungsdaten}

Der Befund erscheint eindeutig. 30 Jahre nach der Wiedervereinigung ist Deutschland noch - oder schon wieder - ein gespaltenes Land. Für diese Einschätzung lassen sich zahlreiche strukturelle Indikatoren ebenso wie aktuelle Daten zu politischen Einstellungen und Verhaltensweise unschwer als Belege finden. Fest steht: Im ost-westdeutschen Vergleich hat Ungleichheit bzw. Unterschiedlichkeit sowohl eine sozial-ökonomische Prägung als auch ein politisch-kulturelles Profil.

Exemplarisch seien hierfür einige wenige aktuelle Fakten in Erinnerung gebracht: Die durchschnittlichen Vermögen liegen im Westen unseres Landes bei durchschnittlich rund 200.000€, im Osten hingegen unter 70.000€ (Grabka 2019, S. 746). Das durchschnittliche ostdeutsche Pro-Kopf-Einkommen lag 2016 bei 84,7\%; 16 Jahre zuvor waren es $81,5 \%$ (WSI 2019). Während die Bruttomonatsverdienste in Westdeutschland 2005 diejenigen in Ostdeutschland um durchschnittlich $770 €$ überstiegen, vergrößerte sich die Differenz bis zum Jahr 2018 auf $844 €$ (Ebenda). Die Arbeitslosenquote betrug 2018 im Osten des Landes 6,9\% gegenüber 4,8\% im Westen (Jahresbericht Dt. Einheit 2019, S. 116). Die Zentralen großer Konzerne sind ganz überwiegend im Westen (464 im Jahr 2016) und selten im Osten (36, einschließlich Berlin) angesiedelt (IWH 2019, S. 37). In ostdeutschen Regionen ist der Anteil von Schulabbrechern signifikant höher (Ebenda, S. 59).

E. Holtmann $(\bowtie)$

Großer Berlin 14, 06108 Halle/Saale, Deutschland

E-Mail: everhard.holtmann@zsh.uni-halle.de

Zentrum für Sozialforschung Halle e.V. (ZSH) an der Martin-Luther-Universität Halle-Wittenberg, Martin-Luther-Universität Halle-Wittenberg, Halle/Saale, Deutschland 
Tab. 1 Wandel ausgewählter politischer Einstellungen in West- und Ostdeutschland 2014-2018 (Quelle: Gabriel 2019; entnommen aus Holtmann 2019, S. 157)

\begin{tabular}{llllllc}
\hline & West & \multicolumn{3}{c}{ Ost } & \multicolumn{3}{c}{ Differenz 2018-2014 } \\
& 2014 & 2018 & 2014 & 2018 & West & Ost \\
\hline Verbundenheit mit dem Wohnort & 77 & 64 & 84 & 72 & -13 & -12 \\
Lokale Integration & 63 & 66 & 61 & 67 & 3 & 6 \\
Politisches Interesse & 45 & 51 & 47 & 52 & 2 & 1 \\
Subjektive politische Kompetenz & 59 & 65 & 55 & 60 & 6 & -5 \\
Präferenz für repräsentative & 39 & 41 & 33 & 29 & 2 & -4 \\
Demokratie & & & & & & -12 \\
Demokratiezufriedenheit & 42 & 39 & 32 & 20 & -3 & -5 \\
Vertrauen zu parteienstaatl. & 42 & 40 & 35 & 30 & -2 & 3 \\
Institutionen & & & & & & 6 \\
Wahrnehmung von Responsivität & 10 & 14 & 10 & 13 & 4 & 5 \\
Vertrauen zu regulativen Institu- & 60 & 65 & 50 & 56 & & \\
tionen & & & & & & \\
\hline
\end{tabular}

Bevölkerungsumfragen Deutschland 2014 und politische Partizipation Ostdeutschland 2018

Angaben: Mittelwerte; die Daten sind jeweils gesondert für West- und Ostdeutschland demographisch gewichtet

Auch im Feld der politischen Einstellungen reproduzieren sich Ost-West-Differenzen ausdauernd. Obschon im zeitlichen Längsschnitt durchaus auch Angleichungstendenzen erkennbar sind, weichen die Zustimmungswerte bei wichtigen Schlüsselindikatoren, die über den Stand der Legitimation des demokratischen Systems Auskunft geben, im Ost-West-Vergleich voneinander ab. So unterschreiten etwa bei der Zufriedenheit mit dem Funktionieren der Demokratie, beim Institutionenvertrauen oder bei der Präferenz für das repräsentative bzw. direkte Demokratiemodell die in Ostdeutschland erhobenen Daten mehr oder minder deutlich die westdeutschen Vergleichswerte (Tab. 1).

Dabei stehen die Daten für „Struktur“ und „Kultur“, also einerseits ökonomische Leistungsfähigkeit, die strukturell eingeschrieben ist in regionale Entwicklungspfade, die unter anderem den ,langen Schatten der DDR-Zentralverwaltungswirtschaft" (IWH 2019, S. 18f.) bis in die Gegenwart werfen, und andererseits eine gegenüber Politik erkennbar größere Skepsis und Distanzhaltung, in einem Zusammenhang. Das gilt jedoch nicht nur für die Ost-West-Differenz. Diese Differenz beschreibt lediglich eine - fraglos gewichtige - Dimension der innerdeutschen Verhältnisse. Wie Tab. 1 veranschaulicht, entwickeln sich manche politischen Einstellungen, wie politisches Interesse und die wahrgenommene Responsivität der Politik, in der Tendenz auch konvergent. Bei der Auswertung neuerer Umfragedaten zur politischen Partizipation zeichnet der Politikwissenschaftler Oscar Gabriel denn auch das Bild einer fortgeschrittenen Angleichung: 30 Jahre nach der Wiedervereinigung ließen sich bezüglich der aktiven politischen Beteiligung keine großen West-Ost-Unterschiede ausmachen. „In der Struktur, im Niveau und in den Bedingungen der politischen Partizipation überwiegen die Gemeinsamkeiten zwischen alten und neuen Bundesländern die Unterschiede" (Gabriel 2019, S. 212).

Und wie die Differenz, ist auch die kulturelle Signatur von Angleichung strukturell unterlegt. Bevor die Produktivität der ostdeutschen Wirtschaft ab etwa dem 
Jahr 2000 „höchstens noch in Trippelschritten“ vorankam und heute bei $82 \%$ des westdeutschen Durchschnitts stagniert, war die Produktivität zunächst schneller als in Westdeutschland gewachsen. Die Lage auf dem Arbeitsmarkt hat sich seit 2005 stetig verbessert. Auch die Nettoabwanderung nach Westdeutschland ist inzwischen zum Stillstand gekommen (Jahresbericht Deutsche Einheit 2019, S. 116; IWH 2019, S. 8 und 48). Bei Bevölkerungsumfragen in den neuen Ländern zeigen sich deutliche Mehrheiten mit ihrer materiellen Situation regelmäßig zufrieden (BrandenburgMonitor 2018, S. 5; Sachsen-Monitor 2018, S. 11, Sachsen-Anhalt-Monitor 2018, S. 35 f., Thüringen-Monitor 2019, S. 97). Das gilt ebenso für die Lebenszufriedenheit. 2016 waren Deutsche in Ost und West im Schnitt zufriedener mit ihrem Leben als jemals zuvor nach der Wiedervereinigung (Jahresbericht Deutsche Einheit 2019, S. 13).

\section{Neue Konfliktmuster und wieder zunehmende Divergenz politischer Einstellungen}

Indes wäre das politische Klima in Ostdeutschland mit den beiden entgegengesetzten Dynamiken von Differenz und Konvergenz immer noch nicht vollständig beschrieben. Hinzu kommt ein Trend zu neuerlicher Divergenz, der in den letzten Jahren sichtbar geworden ist. So ist 2018 die Demokratiezufriedenheit in Ostdeutschland nachgerade dramatisch abgefallen (Tab. 1). Bei Landtagswahlen in den neuen Ländern erzielt die rechtspopulistische AfD seit 2016 überdurchschnittliche Stimmengewinne, die beträchtliche Verschiebungen innerhalb der regionalen Parteiensysteme zur Folge haben. Mit der abrupt aufwachsenden Mobilisierung von Protest und Unzufriedenheit geht, wie wir an anderer Stelle ausgeführt haben, ein folgenreicher Bedeutungswandel demokratischer politischer Partizipation einher. Hierbei ist nicht mehr der Beweggrund vorrangig, mit dem Stimmzettel die Bildung und das Programm einer Regierung zu beeinflussen, sondern bevorzugt geht es darum, Protest auszudrücken (Holtmann 2019, S. 345 f.).

Der politische Wettersturz bei der Zufriedenheit mit dem Zustand der Demokratie und die Radikalisierung im Wahlverhalten zeigen: Die bundesdeutsche Gesellschaft ist, in den Worten des Meinungsforschers Richard Hilmer, gegenwärtig ,,polarisiert wie schon lange nicht mehr". Anders als in den Jahren zuvor, verlaufe die zentrale gesellschaftliche Konfliktlinie ,,aber nicht auf der sozio-ökonomischen, sondern auf der kulturellen Achse“ (Hilmer 2020), d. h. konkreter, wie andere Autoren ausführen, zwischen Gewinnern und (gefühlten) Verlierern von Globalisierung, Entgrenzung und Modernisierung.

\section{3 ,Gewinnerregionen“ und „Schockregionen“ - eine aktuelle innerdeutsche Trennlinie}

Besondere Beachtung verdient Hilmers Hinweis, dass die kulturelle Hauptkonfliktlinie ,nicht primär zwischen Ost und West“" verlaufe, ,,sondern zwischen gesellschaftlichen Gruppen mit unterschiedlichen Einstellungsmustern, die in allen Landesteilen 
zu finden sind" (Ebenda). In dieselbe Richtung weisen auch aktuelle wirtschaftswissenschaftliche Analysen. Zwar blieben, so konstatiert das Leibniz-Institut IWH, die Unterschiede innerhalb Deutschlands auch drei Jahrzehnte nach dem Mauerfall noch sichtbar. Jüngste Ergebnisse der Forschung deuteten jedoch daraufhin, dass „die Bruchkante“ der - in diesem Fall wirtschaftlichen - Entwicklung, ,nicht immer ausschließlich entlang der ehemaligen innerdeutschen Grenze verläuft, sondern neben dem West-Ost-Gefälle auch Süd-Nord- oder Stadt-Land-Unterschiede zutage treten“" (IWH 2019, S. 5).

Tatsächlich bestätigen regional vergleichende Untersuchungen, in welchen Strukturdaten mit Einstellungsdaten auf kreiskommunaler Ebene analytisch verknüpft werden, dass Befragte aus Regionen, die sich entweder günstig oder ungünstig entwickelnde Lebensumfelder bieten, politische Einstellungen hegen, die in Ostwie Westdeutschland in dieselbe Richtung weisen. Ein Erklärungsansatz geht dahin, „Schockregionen“ von „Gewinnerregionen“ klassifikatorisch abzusetzen, also Gegenden zu unterscheiden, die von krisenhaften Entwicklungsverläufen (Wanderungsverlust, Beschäftigungsgrad) unterschiedlich betroffen sind. Der Vergleich auf der Kreisebene ist in mehrfacher Hinsicht aufschlussreich. Zum einen zeigt sich, dass das Einstellungsbild, wie beispielsweise die Demokratiezufriedenheit, die Präferenz für das Modell der repräsentativen Demokratie, das Vertrauen in politische Institutionen, die wahrgenommene Responsivität von Politikern sowie das allgemeine und persönliche Gerechtigkeitsempfinden, in westdeutschen wie ostdeutschen „Schockregionen“ durchgängig negativer ausfällt (exemplarisch hierfür Abb. 1). Diese Trennlinie von innerdeutsch gespaltenen Einstellungsmustern folgt mithin erkennbar nicht dem Verlauf der ehemaligen Grenze zwischen der DDR und der alten Bundesrepublik.

Zum anderen erweist sich, dass die pessimistischen Bewertungen in „Schockregionen" Ostdeutschlands fast ausnahmslos noch markanter sind als in westdeutschen Vergleichsräumen. Schließlich fällt im Osten das Einstellungsgefälle zwischen Schock- und Gewinnerregionen zumeist größer aus als im westdeutschen Vergleich dieser beiden Regionstypen. Das wiederum heißt: West- und Ostdeutsche reagieren
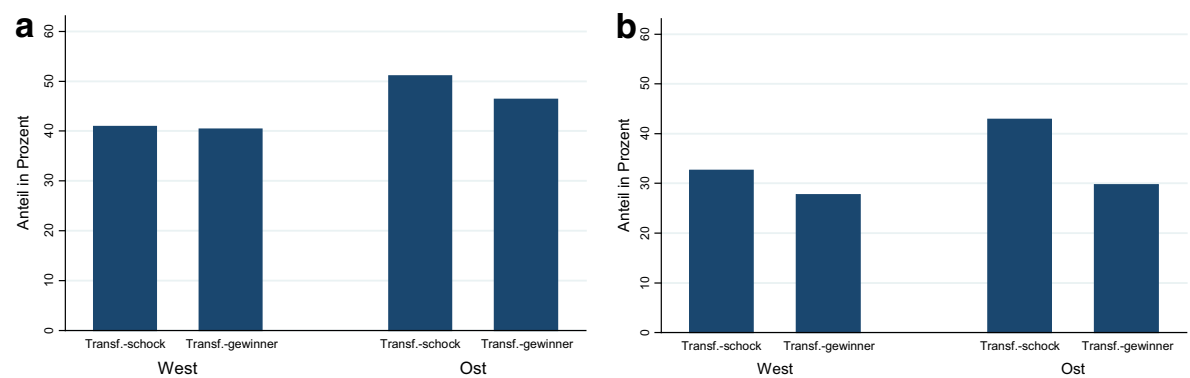

Abb. 1 Gerechtigkeitsempfinden nach Gewinner- und Schockregionen in Ost- und Westdeutschland. a Geht es Deutschland eher gerecht oder eher ungerecht zu? - Eher ungerecht nach Transformationsschock in Ost- und Westdeutschland. b Erhalten Sie persönlich den gerechten Anteil? - Etwas/sehr viel weniger nach Wahlpräferenz in in Ost- und Westdeutschland. (Quelle: Brachert 2020, Berechnungen auf der Basis der Bevölkerungsumfrage Politische Partizipation Ostdeutschland 2018 - entnommen aus Holtmann 2019, S. 271) 


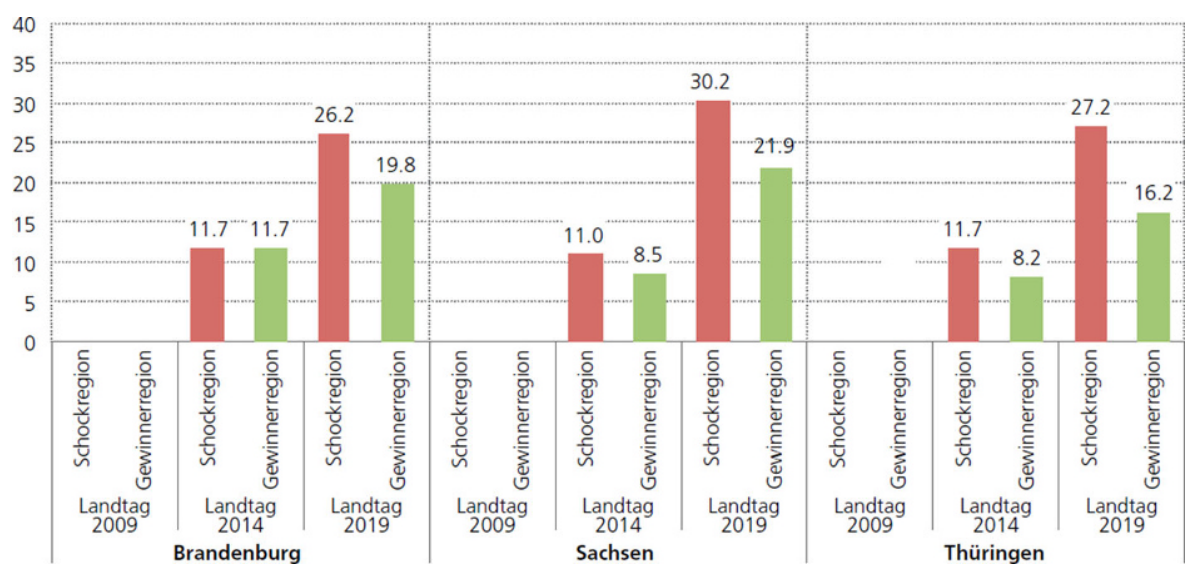

Abb. 2 AfD-Stimmenanteile bei ostdeutschen Landtagswahlen 2019 nach Gewinner- und Schockregionen (Angaben in Prozent). (Eigene Berechnungen auf der Basis der Daten des Bundeswahlleiters - entnommen aus Brachert et al. 2020, S. 26)

in ihren politischen Einstellungen auf krisenhafte Lebensbedingungen zwar ähnlich sensibel, aber unterschiedlich intensiv. Der von den Autoren dieser Untersuchung so apostrophierte „doppelte Transformationsschock“ mit dem die ostdeutsche Bevölkerung zunächst während der 1990er Jahre und dann in der Finanz- und Wirtschaftskrise 2008/09 konfrontiert wurde, ist weiterhin bewusstseinsbildend. Diese Schockerfahrung wirkt nach als ein psychologischer Hintergrundfaktor, welcher die in Ostdeutschland aktuell größeren Legitimationsdefizite des demokratischen Systems mindestens zum Teil erklären kann und sich in einer im östlichen Landesteil höheren Wähler*innensympathie für die AfD niederschlägt (Abb. 2).

Das Zwischenfazit lautet: Ungeachtet langfristiger Angleichungstendenzen sind Ost- und Westdeutschland 30 Jahre nach der Wiedervereinigung auf der Ebene der politischen Einstellungen nachweislich gespalten. Doch in gesamtdeutscher Perspektive gibt es eine doppelte Differenz: In Ost- und Westdeutschland treten regionale Unterschiede hervor, welche prekäre Lebensumfelder widerspiegeln, die hier wie dort existieren und das eingeschliffene Ost-West-Trennmuster unterlaufen.

\section{Die Ost-West-Differenz als Moralfrage}

Allein mit den Effekten des „doppelten Transformationsschocks“ ist die in Ostdeutschland besonders ausgeprägte Politik- und Gesellschaftskritik im Übrigen nicht hinreichend erklärt. Hinzu kommt, dass im Osten des Landes Erscheinungsformen von strukturell verfestigter Ungleichheit vergleichsweise stark zu Moralfragen werden. Beispielhaft hierfür ist das bis heute mehr als im Westen verbreitete Empfinden, allgemein und persönlich nicht gerecht behandelt zu werden (Abb. 1). Immer noch betrachten sich bei Umfragen Ostdeutsche mehrheitlich als „Bürger zweiter Klasse“ (Jahresbericht Deutsche Einheit 2019, S. 13). Das latente Grundgefühl von Benachteiligung hält sich ausdauernd seit den 1990er-Jahren, kontrastierend zur ganz 
überwiegend positiven Einschätzung der eigenen materiellen Lebenslage und deren sozialer Einbettung. Die Empfindlichkeit bietet einen Resonanzkörper für öffentliche Debatten etwa um „die Treuhand“ oder die Unterrepräsentanz Ostdeutscher in bundesdeutschen Eliten. Wo dieser historisch wie aktuell notwendige Diskurs moralisch aufgeladen wird, finden wissenschaftliche Darstellungen, die diese Themen differenziert behandeln (Böick 2020; Kollmorgen 2020), weniger Gehör.

\section{Ist das Konstrukt einer ,ostdeutschen Identität“" hilfreich? - Schlussbemerkung}

Wie lässt sich die Ost-West-Differenz ehestens überwinden? - Hierzu einige kurze Anmerkungen. Zunächst einmal dürfte grundsätzliches Einvernehmen darüber bestehen, dass „Differenz“ im Vergleich von Ost- und Westdeutschland nicht per se korrekturbedürftig ist, sondern vielmehr auch eine Normallage moderner und sich dynamisch entwickelnder - eben in sich differenzierter - Staatsgesellschaften abbildet. Dass gewachsene landesgeschichtliche Traditionen sowie regionale und lokale Vielfalt von gruppenbezogenen Identitäten und individuellen Lebensentwürfen etwas Positives und Erhaltenswertes darstellen, wird kaum jemand ernsthaft in Frage stellen wollen.

Andererseits verweist die ausgeprägt negative Bewertung, die Politik und Politikern in strukturell benachteiligten Regionen widerfährt, auf objektiv gegebene Defizite räumlicher Gerechtigkeit, die ländliche Räume zwar nicht ausschließlich im Osten Deutschlands, aber besonders dort kennzeichnet. Diesen Disparitäten, die von den betroffenen Menschen als Ausdünnung von Infrastrukturangeboten und als Unterversorgung mit öffentlichen Gütern erfahren werden, sollte mittels einer Strukturpolitik gegengesteuert werden, die sich gerade nicht vornehmlich auf städtische Mittelpunkte konzentriert. Gleichwertige Lebensverhältnisse herzustellen ist eine gesamtdeutsche Aufgabe, wobei nicht (mehr) ,der Osten“ gegenüber ,,dem Westen" in Stellung gebracht, sondern vergleichbare Problemräume kleinen Formats im Osten und im Westen gleichermaßen aufgewertet werden sollten.

Unter der Voraussetzung, dass diese nationale Kraftanstrengung effektiv vorangebracht wird, bedarf es nicht einer von östlicher Seite vermeintlich immer nötigen „Selbstbehauptung gegen den Westen“, wie sie die Journalistin Anne Haehnig in ihrem im Mai 2020 in der ZEIT erschienenen Beitrag einfordert (Hähnig 2020). Das Reklamieren einer eigenen ostdeutschen Identität, also des abgrenzenden Zusammenschlusses nach innen, könnte zur Folge haben, dass die Ost-West-Spaltung wieder vertieft wird. Diese Differenz aber, die für viele vor allem jüngere Ostdeutsche längst keine Bedeutung mehr hat, sollte nicht künstlich beatmet werden.

Funding Open Access funding provided by Projekt DEAL.

Open Access Dieser Artikel wird unter der Creative Commons Namensnennung 4.0 International Lizenz veröffentlicht, welche die Nutzung, Vervielfältigung, Bearbeitung, Verbreitung und Wiedergabe in jeglichem Medium und Format erlaubt, sofern Sie den/die ursprünglichen Autor(en) und die Quelle ordnungsgemäß nennen, einen Link zur Creative Commons Lizenz beifügen und angeben, ob Änderungen vorgenommen wurden. 
Die in diesem Artikel enthaltenen Bilder und sonstiges Drittmaterial unterliegen ebenfalls der genannten Creative Commons Lizenz, sofern sich aus der Abbildungslegende nichts anderes ergibt. Sofern das betreffende Material nicht unter der genannten Creative Commons Lizenz steht und die betreffende Handlung nicht nach gesetzlichen Vorschriften erlaubt ist, ist für die oben aufgeführten Weiterverwendungen des Materials die Einwilligung des jeweiligen Rechteinhabers einzuholen.

Weitere Details zur Lizenz entnehmen Sie bitte der Lizenzinformation auf http://creativecommons.org/ licenses/by/4.0/deed.de.

\section{Literatur}

2018. Brandenburg-Monitor. https://www.brandenburg.de/media/bb1.a.3780.de/bb_monitor.pdf. Zugegriffen: 6. Juni 2020.

2018. Sachsen-Anhalt-Monitor. https://www.staatsregierung.sachsen.de/sachsen-monitor-5656.html. Zugegriffen: 6. Juni 2020.

2018. Sachsen-Monitor. https://www.staatsregierung.sachsen.de/sachsen-monitor-2018-5616.html. Zugegriffen: 6. Juni 2020

2019. Thüringen-Monitor. https://www.landesregierung-thueringen.de/regierung/th-monitor. Zugegriffen: 6. Juni 2020

Böick, Markus. 2020. Treuhandanstalt und Wirtschaftsumbau. Dossier „Lange Wege der deutschen Einigung" der Bundeszentrale für politische Bildung. https://www.bpb.de/geschichte/deutsche-einheit/ lange-wege-der-deutschen-einheit/. Zugegriffen: 6. Juni 2020

Brachert, Matthias, Everhard Holtmann, und Tobias Jaeck. 2020. Einflüsse des Lebensumfelds auf politische Einstellungen und Wahlverhalten. Eine vergleichende Analyse der Landtagswahlen 2019 in drei ostdeutschen Bundesländern. FES Empirische Sozialforschung, Bd. 12. Berlin: Friedrich-EbertStiftung.

Brachert, Matthias. 2020. Bestimmungsgründe regionaler Unterschiede der politischen Partizipation in Deutschland. In Die Umdeutung der Demokratie. Politische Partizipation in Ost- und Westdeutschland, Hrsg. Everhard Holtmann, 219-290. Frankfurt/ New York:Campus

Bundesregierung. Jahresbericht der Bundesregierung zum Stand der Deutschen Einheit 2019. www. beauftragter-neue-laender.de. Zugegriffen: 6. Juni 2020

Gabriel, Oscar W. 2019. Politische Partizipation im ausgehenden dritten Jahrzehnt des vereinigten Deutschland. In Die Umdeutung der Demokratie. Politische Partizipation in Ost- und Westdeutschland, Hrsg. Everhard Holtmann, 143-217. Frankfurt/ New York: Campus.

Grabka, Markus M. 2019. Die Vermögensbildung in Deutschland ist dringend reformbedürftig. DIW-Wochenbericht 40:746.

Hähnig, Anne. 2020. Der Osten braucht eine neue Elite! Die Zeit im Osten 23. 28. Mai 2020.

Hilmer, Richard. 2020. Politische Einheit, gespaltene Meinungsmuster. Online-Dossier der Bundeszentrale für politische Bildung: Die langen Wege der deutschen Einigung. Dossier „Lange Wege der deutschen Einigung" der Bundeszentrale für politische Bildung. https://www.bpb.de/geschichte/deutscheeinheit/lange-wege-der-deutschen-einheit/ (forthcoming).

Holtmann, Everhard (Hrsg.). 2019. Die Umdeutung der Demokratie. Politische Partizipation in Ost- und Westdeutschland. Frankfurt/ New York: Campus.

Institut für Wirtschaftsforschung Halle (IWH). 2019. Vereintes Land - drei Jahrzehnte nach dem Mauerfall. https://www.iwh-halle.de/fileadmin/user_upload/publications/sonstint/2019_iwh_vereintesland_de.pdf. Zugegriffen: 10. Juni 2020.

Kollmorgen, Raj. 2020. Zwischen Austausch und Marginalisierung. Ostdeutsche in den Eliten nach der Vereinigung. Dossier „Lange Wege der deutschen Einigung“ der Bundeszentrale für politische Bildung. https://www.bpb.de/geschichte/deutsche-einheit/lange-wege-der-deutschen-einheit/. Zugegriffen: 6. Juni 2020

WSI. 2019. WSI-Verteilungsmonitor Stand April. https://www.wsi.de/de/wsi-verteilungsmonitor-14559. htm. Zugegriffen: 10. Juni 2020. 